\title{
Return of the prodigal Goldstone boson
}

\author{
J. Kapusta \\ School of Physics and Astronomy, University of Minnesota, Minneapolis, Minnesota 55455 \\ D. Kharzeev \\ Theory Division, CERN, Geneva, Switzerland \\ and Fakultät für Physik, Universtät Bielefeld, Bielefeld, Germany \\ L. McLerran \\ School of Physics and Astronomy, University of Minnesota, Minneapolis, Minnesota 55455
}

(Received 14 July 1995)

\begin{abstract}
We propose that the mass of the $\eta^{\prime}$ meson is a particularly sensitive probe of the properties of finite energy density hadronic matter and quark-gluon plasma. We argue that the mass of the $\eta^{\prime}$ excitation in hot and dense matter should be small, and, therefore, that the $\eta^{\prime}$ production cross section should be much increased relative to that for $p p$ collisions. This may have observable consequences in dilepton and diphoton experiments.
\end{abstract}

PACS number(s): 11.30.Rd, 12.38.Mh, 24.85.+p, 25.75.- $\mathrm{q}$

\section{INTRODUCTION}

One of the great mysteries of the quark model is why there is no ninth Goldstone boson whose mass is somewhere between that of the pion and that of the kaon. Roughly stated, the problem is that in the limit of massless quarks, the quark model has a U(3) chiral symmetry. This chiral symmetry, when broken, predicts the existence of nine massless Goldstone bosons. In nature, however, there are only eight light mesons.

The problem is resolved by the Adler-Bell-Jackiw U(1) anomaly [1-3]: the $U(1)$ part of the $U(3)$ symmetry is explicitly broken by interactions. It is possible to show explicitly that instantons [4,5] dynamically induce the $U(1)$ chiral symmetry breaking. This results in giving the ninth Goldstone boson a mass. The would be ninth Goldstone boson is presumably the $\eta^{\prime}$, which has a mass of nearly one $\mathrm{GeV}$.

As the density of matter is increased, it is expected that the effects induced by the tunneling between different topological vacua of QCD will rapidly disappear [6-11]. Let us briefly recall the origin of this belief, based on the example of the instanton solution realizing this tunneling. The amplitude $\mathscr{T}$ of the tunneling transition, calculated in the quasiclassical approximation of instanton configurations, is

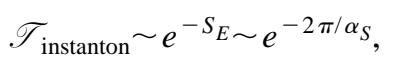

where $S_{E}$ is the Euclidean action of the instanton solution. It is expected that the effects of finite energy density will make $\alpha_{S}$ density dependent such that, for large energy densities,

$$
\alpha_{S} \sim \frac{24 \pi}{\left(33-2 N_{f}\right) \ln \left(\epsilon / \Lambda^{4}\right)},
$$

where $\epsilon$ is the energy density and $\Lambda \sim 200 \mathrm{MeV}$. As the energy density increases, the effects of instantons rapidly decrease. Note that $\Lambda^{4} \sim 200 \mathrm{MeV} / \mathrm{fm}^{3}$ is a relatively low energy density. (Recent computations of the instanton density as a function of temperature have been given in Refs. $[12,13]$.)

We therefore expect that as the energy density of hadronic matter is increased, the mass of the $\eta^{\prime}$ will be a rapidly falling function of energy density. In the quark-gluon plasma, we expect that excitations with the quantum numbers of the $\eta^{\prime}$ will become almost mass degenerate, modulo current quark mass corrections, with excitations with quantum numbers of the octet of pseudoscalar Goldstone bosons. This is manifest in the quark model since there will be no penalty for making an isospin singlet configuration of quarks relative to an isotriplet configuration.

The plan of this paper is as follows. In Sec. II we recall the mechanism responsible for the large mass of the $\eta^{\prime}$ in QCD, and argue about the properties of the $\eta^{\prime}$ at high densities. In Sec. III we discuss the dynamics of $\eta^{\prime}$ production and annihilation in hot and dense matter. In Sec. IV we discuss several expected signals of the proposed enhancement of $\eta^{\prime}$ production in dense matter and claim possible experimental evidence in favor of our scenario.

\section{AXIAL ANOMALY, GHOST, AND $\eta^{\prime}$ AT HIGH DENSITIES}

Consider a quark-antiquark pseudoscalar flavor-singlet field

$$
\left|\eta_{0}\right\rangle=\frac{1}{\sqrt{3}}|\bar{u} u+\bar{d} d+\overline{s s}\rangle
$$

The divergence of the corresponding flavor-singlet axial vector current acquires an anomalous part, due to the interaction with gluon fields, which does not disappear in the chiral limit $m \rightarrow 0$ of massless quarks:

$$
\partial^{\mu} J_{5 \mu}^{0}=2 i \sum_{f} m_{f} \bar{q}_{f} \gamma_{5} q_{f}+2 N_{f} \frac{g^{2}}{16 \pi^{2}} \operatorname{Tr}\left(G_{\mu \nu} \widetilde{G}^{\mu \nu}\right)
$$


This anomalous part may be written as the full divergence of the gauge-dependent topological current

$$
K_{\mu}=2 N_{f} \frac{g^{2}}{16 \pi^{2}} \epsilon_{\mu \nu \lambda \rho} \operatorname{Tr}\left(G^{\nu \lambda} A^{\rho}\right),
$$

so that in the chiral limit one has the Adler-Bardeen relation

$$
\partial^{\mu} J_{5 \mu}^{0}=\partial^{\mu} K_{\mu} .
$$

It is possible to introduce a new axial vector current

$$
J_{5 \mu}=J_{5 \mu}^{0}-K_{\mu},
$$

which is explicitly conserved in the chiral limit:

$$
\partial^{\mu} J_{5 \mu}=2 i \sum_{f} m_{f} \bar{q}_{f} \gamma_{5} q_{f}
$$

The corresponding charge

$$
Q_{5}=\int d^{3} x J_{50}
$$

is naively expected to be conserved. Since this charge is the generator of the $\mathrm{U}(1)_{A}$ symmetry, and this symmetry is not observed in the hadron spectrum (no parity doublet exists), we have to presume that the symmetry is spontaneously broken. This would lead to the appearance of a nearly massless Goldstone boson field (3). In nature, however, the physical $\eta^{\prime}$ meson has a large mass of almost one $\mathrm{GeV}$ and therefore cannot be considered a Goldstone boson.

To check if the charge (9) is really conserved, one can integrate the divergence of the current $J_{5 \mu}$ over Euclidean four-space. After the spatial integration is performed, the result can be represented as

$$
\int_{-\infty}^{+\infty} d t \frac{d Q_{5}}{d t}=2 N_{f} \nu[G],
$$

where

$$
\nu[G]=2 N_{f} \frac{g^{2}}{32 \pi^{2}} \int d^{4} x \operatorname{Tr}\left(G_{\mu \nu} \widetilde{G}^{\mu \nu}\right)
$$

is the so-called topological charge. It is equal to zero in Abelian theories, but in QCD $\nu[G] \neq 0$ : the one-instanton solution, for example, yields $\nu[G]=1$. Therefore the charge (9) is not a conserved quantity, and going from (Euclidean) $t=-\infty$ to $t=+\infty$ it changes by

$$
\Delta Q_{5}=2 N_{f} \nu[G]
$$

Therefore, the existence of nontrivial topological solutions explicitly breaks the $\mathrm{U}(1)_{A}$ symmetry, resulting in the vanishing of the corresponding Goldstone mode.

As we have already mentioned in the Introduction, the instanton density vanishes in the high energy density limit as $g^{2}=4 \pi \alpha_{S} \rightarrow 0$. We therefore expect that in dense matter the ensemble-averaged axial charge $Q_{5}$ will be conserved:

$$
\frac{d\left\langle Q_{5}\right\rangle}{d t}=0
$$

If the $\mathrm{U}(1)_{A}$ symmetry is still spontaneously broken at very high densities, it would imply the return of the ninth Goldstone boson.

Even though the arguments presented above explain on a qualitative level why the physical $\eta^{\prime}$ is not a Goldstone excitation, and under what circumstances can it again become one, it is instructive for our purposes to establish the actual relation between the properties of the vacuum and the mass of the $\eta^{\prime}$. To do this we follow the approach developed by Witten [14] and Veneziano [15]. They noticed that the nonvanishing of the topological charge (11) implies the existence of an unphysical massless pole, introduced earlier by Kogut and Susskind [16], in the correlator of the topological current (5). Such a pole means the existence of a massless excitation, or "ghost," which should reflect some fundamental symmetry of the theory. As was shown by Dyakonov and Eides [17], the origin of this excitation in QCD is the periodicity of the potential energy of the vacuum with respect to the collective coordinate:

$$
X=\int d^{3} x K_{0}(x)
$$

The potential barriers separating different vacua are penetrable, by instantons for example, and the massless ghost just corresponds to this degree of freedom in the theory. If one introduces the propagator $\left\langle a_{\alpha} a_{\beta}\right\rangle$ of the ghost field $a_{\alpha}$, the residue of the ghost contribution $\lambda$ can be defined as

$$
\left\langle 0\left|K_{\alpha} K_{\beta}\right| 0\right\rangle=\lambda^{4}\left\langle 0\left|a_{\alpha} a_{\beta}\right| 0\right\rangle
$$

so that, as $q^{2} \rightarrow 0$,

$$
q_{\alpha} q_{\beta}\left\langle 0\left|K_{\alpha} K_{\beta}\right| 0\right\rangle=\langle 0|\nu \nu| 0\rangle=-\lambda^{4} \neq 0 .
$$

Note that, apart from the ghost contribution, the propagator of the topological current also contains the normal gluon part.

The field (3) can now mix with the ghost, the amplitude of mixing being of order $\lambda^{2} / f_{\eta^{\prime}}$, where $f_{\eta^{\prime}}$ is the $\eta^{\prime}$ decay constant. As a result of this mixing the physical $\eta^{\prime}$ acquires an additional mass

$$
\Delta m \simeq \lambda^{2} / f_{\eta^{\prime}}
$$

so that the mass of the $\eta^{\prime}$ does not vanish in the chiral limit:

$$
m_{\eta^{\prime}}^{2}=m_{0}^{2}+(\Delta m)^{2} .
$$

The mass of the bare $\eta^{\prime}$ field (3) can simply be estimated in the free quark model as

$$
m_{0}^{2}=\frac{1}{3}\left(2 m_{K}^{2}+m_{\pi}^{2}\right)
$$

At high energy densities we expect that the density of instantons will diminish, the ghosts will disappear, and the $\eta^{\prime}$ will be (almost) entirely described by the field (3), whose mass will then be given by (19) and equal to $m_{0} \simeq 400 \mathrm{MeV}$.

Of course, in nature the situation is likely to be a bit more complicated. Indeed, the mass eigenstates in the isosinglet channel are not the $\eta$ and $\eta^{\prime}$, but the nonstrange and strange states $\left|\eta_{\mathrm{NS}}\right\rangle=|\bar{u} u+\bar{d} d\rangle / \sqrt{2}$ and $\left|\eta_{\mathrm{S}}\right\rangle=|\overline{s s}\rangle$. These states 
can only mix if one allows for intermediate gluon states. The extreme assumption that the only allowed gluonic states are nonperturbative ghostlike states would lead to the conclusion that at high densities, when ghosts disappear, the physical isosinglet excitations will be $\eta_{\mathrm{NS}}$ and $\eta_{\mathrm{S}}$. Their masses will then be $m_{\mathrm{NS}}^{2}=m_{\pi}^{2}$ and $m_{\mathrm{S}}^{2}=2 m_{K}^{2}-m_{\pi}^{2} ; m_{\mathrm{S}} \simeq 700 \mathrm{MeV}$. However, normal gluonic states certainly contribute, and we expect that the states $\eta_{\mathrm{NS}}$ and $\eta_{\mathrm{S}}$ will mix even at high densities, even though this mixing will probably not yield the states with the $\eta$ and $\eta^{\prime}$ quark wave functions. We expect also that as a consequence of the effects discussed above, the $\eta-\eta^{\prime}$ mixing will be strongly dependent on energy density, and the physical $\eta$ mass will decrease too. Nevertheless, since the topological and perturbative gluonic effects are very difficult to separate, for the sake of argument we will assume in the rest of this paper that the $\eta^{\prime}$ quark content at any density is given by (3).

\section{DYNAMICS}

Production cross sections for light mesons are typically of the order predicted by the Hagedorn model,

$$
\sigma_{i} \sim g_{i}(M / 2 \pi)^{3 / 2} e^{-M / T_{H}}
$$

when the particle mass is large compared to $T_{H} \sim 160 \mathrm{MeV}$. The quantity $g_{i}$ is the number of internal degrees of freedom of the $i$ th particle species. For pions this same model gives

$$
\sigma_{\pi} \sim g_{i} / \pi^{2}
$$

Using this rather simple model we see that the expected cross section of $\eta^{\prime}$ production is quite small, $\sigma_{\eta^{\prime}} / \sigma_{\pi^{0}}$ $\sim 2 \times 10^{-2}$.

Now, suppose that the $\eta^{\prime}$ is made in a dense environment. Here, we expect that the mass of the $\eta^{\prime}$ is small, and the particle ratio $N_{\eta^{\prime}} / N_{\pi^{0}} \sim 1$. If the $\eta^{\prime}$ becomes a Goldstone boson we might get an enhancement of up to 50 in the production cross section. This should of course be considered only as an absolute upper bound for the enhancement; the strange quark mass effects [see (19)] result in a more moderate enhancement factor of 16 , and if the $\eta^{\prime}$ at high densities becomes an $|\overline{s s}\rangle$ state according to the scenario described at the end of the previous section, the enhancement factor will be equal to a relatively modest value of 3 .

After an $\eta^{\prime}$ is produced it must survive subsequent hadronic interactions until it has escaped the matter. The $\eta^{\prime}$ lifetime in vacuum is about $1000 \mathrm{fm} / c$; if there was no interaction with surrounding particles, it would certainly survive the time it takes for the hadronic matter produced in heavy ion collisions to dissipate.

It is amazing that the results presented in the previous section imply that the $\eta^{\prime}$ should decouple from high density matter and therefore most likely cannot be absorbed. To see this, we will follow the line of reasoning developed in Refs. $[18,19]$.

Let us first note that the Adler-Bardeen relation (6), and an analogue of the PCAC (partial conservation of axial vector current) for the $\eta^{\prime}$ field,

$$
\eta^{\prime}(x)=\frac{1}{m_{\eta^{\prime}}^{2} f_{\eta^{\prime}}^{2}} \partial^{\mu} J_{5 \mu}^{0},
$$

suggest the existence of a relation between the matrix elements of the $\eta^{\prime}$ field and of the topological charge (11). With this in mind, we consider a nonsymmetric matrix element of the topological current (5) between some hadronic states. ${ }^{1}$ For definiteness, we consider nucleons explicitly here. It has the general form

$$
\left\langle p^{\prime}\left|K_{\nu}\right| p\right\rangle=\bar{u}\left(p^{\prime}\right)\left[\gamma_{\nu} \gamma_{5} G_{1}\left(q^{2}\right)+q_{\nu} \gamma_{5} G_{2}\left(q^{2}\right)\right] u(p),
$$

where $q=p-p^{\prime}, \bar{u}, u$ are the nucleon wave functions, and $G_{1,2}$ are the form factors. Consider the matrix element $\left\langle 0\left|\partial^{\nu} K_{\nu}\right| \bar{N} N\right\rangle$ in the cross channel. Saturating it by the $\eta^{\prime}$ pole, one obtains

$$
q^{2} G_{2}\left(q^{2}\right)=\left\langle 0|\nu| \eta^{\prime}\right\rangle \frac{1}{q^{2}-m_{\eta^{\prime}}^{2}}\left\langle\eta^{\prime} \mid \bar{N} N\right\rangle
$$

where the last matrix element is just the $\eta^{\prime}$ coupling constant $g_{\eta^{\prime} N N}$. The first matrix element can be evaluated by using the Lehmann-Symanzik-Zimmerman reduction formula in the form

$$
\begin{aligned}
\left\langle 0|\nu| \eta^{\prime}\right\rangle & =\int d^{4} x e^{i q \cdot x}\left(-\partial_{x}^{4}+m^{2}\right)\left\langle 0\left|T\left\{\nu \eta^{\prime}(x)\right\}\right| 0\right\rangle \\
& =-\frac{-q^{2}+m_{\eta^{\prime}}^{2}}{m_{\eta^{\prime}}^{2} f^{2}}\langle 0|T\{\nu \nu\}| 0\rangle .
\end{aligned}
$$

As $q^{2} \rightarrow 0$ we get, from (24), (25), and (16), that

$$
q^{2} G_{2}\left(q^{2}\right) \sim \frac{\lambda^{4} g_{\eta^{\prime} N N}}{m_{\eta^{\prime}}^{2} f_{\eta^{\prime}}}=f_{\eta^{\prime}} g_{\eta^{\prime} N N},
$$

where at the last step we used the relation $m_{\eta^{\prime}} \simeq \lambda^{2} / f_{\eta^{\prime}}$, valid in the chiral limit [see (17) and (18)].

In the absence of ghosts, which we expect is the case in high density matter, the form factor $G_{2}\left(q^{2}\right)$ does not possess a zero-mass pole, and the left-hand side of (26) is equal to zero at $q^{2}=0$. Therefore, since $f_{\eta^{\prime}} \neq 0$, we are led to the conclusion that at high densities, the coupling of the $\eta^{\prime}$ vanishes and it decouples from (non-Goldstone-like) matter. A parallel discussion for the coupling of an $\eta^{\prime}$ with two $\rho$ mesons [20] can be given with a similar conclusion.

In the above analysis, we have neglected the contribution arising from the first term on the right-hand side (23). Including this term leads to a Goldberger-Treiman relation of the form $g_{\eta^{\prime} N N} f_{\eta^{\prime}}=g_{A}^{0} m_{N}$ if evaluated between nucleon states in vacuum, even if the topological current were conserved. Here, we will assume that the temperature is sufficently large so that chiral symmetry is restored. In this case, the matrix element can be evaluated perturbatively, and is proportional to quark masses, which we shall take to be small. Of course,

\footnotetext{
${ }^{1}$ In principle, one can consider the matrix elements taken over the ensemble as a whole.
} 
the strange quark mass is not entirely negligible at realistic temperatures achievable in heavy ion collisions, and the $\eta^{\prime}$ will not entirely decouple.

Next, consider moderate to low energy density matter where pions are the most abundant constituents. Then, we need to know the cross section for the annihilation reaction $\pi^{+}+\eta^{\prime} \rightarrow \pi^{+}+\rho^{0}$, which is exothermic, and the isospinrelated cross sections. The rate can be calculated in the low temperature limit using a low energy effective Lagrangian.

The cross section for $\pi\left(p_{1}\right)+\eta^{\prime}\left(p_{2}\right) \rightarrow \pi\left(p_{1}^{\prime}\right)+\rho\left(p_{2}^{\prime}\right)$ is dominated by the exchange of a $\rho$ meson in the $t$ channel. (There are of course contributions from other channels which may become increasingly important at higher temperatures, and this estimate must therefore be regarded as a lower bound on the interaction cross section.) The $\rho \pi \pi$ vertex is well known, and the $\eta^{\prime} \rho \rho$ vertex is the anomalous one $[21,22]$. The matrix element is

$$
\begin{aligned}
\mathscr{L}= & g_{\eta^{\prime} \rho \rho} p_{2 \alpha} p_{2 \beta}^{\prime} \epsilon^{\alpha \beta \nu \tau}\left[-\frac{g_{\mu \nu}}{q^{2}-m_{\rho}^{2}}+\frac{q_{\mu} q_{\nu}}{\left(q^{2}-m_{\rho}^{2}\right) m_{\rho}^{2}}\right] \\
& \times g_{\rho \pi \pi}\left(p_{1}+p_{1}^{\prime}\right)^{\mu} \varepsilon_{\tau}\left(p_{2}^{\prime}\right),
\end{aligned}
$$

where $q=p_{1}^{\prime}-p_{1}$. The total cross section for one-charge configuration works out to be

$$
\begin{aligned}
\sigma_{0}(s)= & \frac{g_{\rho \pi \pi}^{2} g_{\eta^{\prime} \rho \rho}^{2}}{16 \pi p_{c m}^{2}}\left\{\left(t_{+}-t_{-}\right)+\left(t_{+}+t_{-}-2 m_{\rho}^{2}\right)\right. \\
& \times \ln \left(\frac{m_{\rho}^{2}-t_{-}}{m_{\rho}^{2}-t_{+}}\right)+\frac{\left(t_{+}-t_{-}\right)}{\left(m_{\rho}^{2}-t_{-}\right)\left(m_{\rho}^{2}-t_{+}\right)} \\
& \left.\times\left[-m_{\rho}^{2}\left(t_{+}+t_{-}\right)+m_{\rho}^{4}+m_{\pi}^{2}\left(m_{\eta^{\prime}}^{2}-m_{\rho}^{2}\right)^{2} / s\right]\right\} .
\end{aligned}
$$

Here $t_{+}$and $t_{-}$are the kinematic limits of $t$.

From the decay rate for $\rho \rightarrow \pi \pi$, we know that $g_{\rho \pi \pi}^{2} / 4 \pi$ $=2.90$. From the decay rate for $\eta^{\prime} \rightarrow \rho \gamma,[23]$ together with vector meson dominance [21,22], we get $g_{\eta^{\prime} \rho \rho}=3.96 \times$ $10^{-3} / \mathrm{MeV}$ or, more usefully, $g_{\eta^{\prime} \rho \rho}^{2}=6.10 \mathrm{mb}$. It may be noted that this value is consistent with that predicted by gauging the Wess-Zumino term, which is

$$
g_{\eta^{\prime} \rho \rho}=\frac{g_{\rho \pi \pi}^{2}}{16 \pi^{2} f_{\pi}}\left(\sqrt{6} \cos \theta_{P}+\sqrt{3} \sin \theta_{P}\right),
$$

where $\theta_{P}$ is a pseudoscalar mixing angle with a value of about $-20 \pm 5$ degrees [21,22,24].

The annihilation cross section vanishes at threshold and rises monotonically with $s$. Although thermal averaging can be done numerically to obtain the rate, we shall be content with the following simple estimate. For a collision between an $\eta^{\prime}$ and a pion the average value of $s$ at temperature $T$ is easily found to be

$$
\langle s\rangle=\left(m_{\eta^{\prime}}+m_{\pi}\right)^{2}+6 m_{\eta^{\prime}} T .
$$

At $T=150 \mathrm{MeV}, \sqrt{\langle s\rangle}=1.44 \mathrm{GeV}$. At this value, $\sigma_{0}=2.6$ $\mathrm{mb}$. The mean free path $l$ for $\eta^{\prime}$ annihilation is estimated from

$$
l^{-1}=\sum_{i j} \sigma_{i j} n_{i}=2 \sigma_{0} n
$$

where the sum is over all channels, $n$ is the total pion number density, and $\sigma_{0}$ is evaluated at the average $\sqrt{s}$. For temperatures comparable to or greater than the pion mass, the number density is approximately $0.365 T^{3}$. At $T=150 \mathrm{MeV}$, the mean free path for annihilation is $12 \mathrm{fm}$. It gets even bigger as the temperature decreases. Since the $\eta^{\prime}$ decouples near the phase transition temperature, where the present estimate is not valid, we may conclude that $\eta^{\prime}$ s will not annihilate to any appreciable degree at any temperature during the expansion.

It might seem paradoxical to argue that the $\eta^{\prime}$ decouples at high density yet is produced in roughly equal numbers as the pion. Actually, there is no paradox. Suppose that quarkgluon plasma is formed initially. When it hadronizes, all Goldstone bosons will be produced in roughly equal numbers by condensation of the quark and gluon fields. Suppose that high density hadronic matter is formed initially, not the quark-gluon plasma. Then the initial state is formed via meson production in elementary nucleon-nucleon collisions. Many pions will be produced. In this environment, the $\eta^{\prime}$ mass will be low. Since there is no suppression of transitions

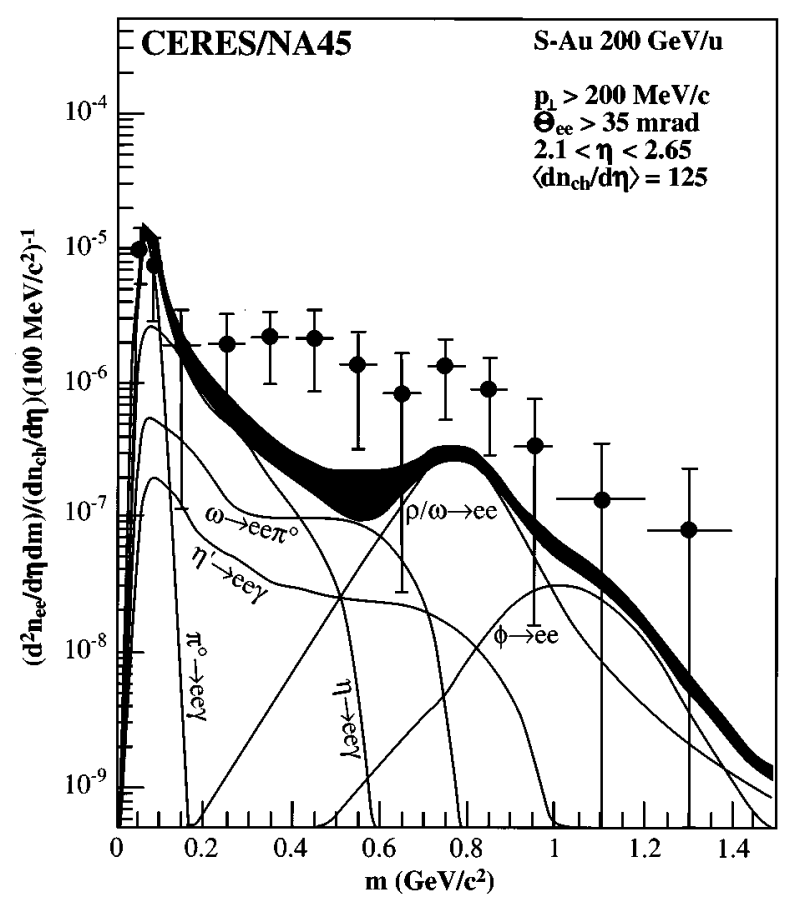

FIG. 1. Yield of low mass dileptons as measured by CERES [25]. Included in the plot are their assumed resonance contributions. The heavy shaded area is the result of summing all these contributions, including estimated uncertainties. 
among the Goldstone bosons themselves, the $\eta^{\prime}$ mesons will come to, or at least approach, chemical equilibrium with pions, kaons, and $\eta$ mesons.

\section{SIGNALS}

During the expansion and cooling phase, the $\eta^{\prime}$ propagates in the background field of the surrounding hadronic matter. This background field increases the $\eta^{\prime}$ mass as the hadronic matter becomes more dilute. Because of energy conservation, any motion of the $\eta^{\prime}$ relative to this medium will be damped, and the $\eta^{\prime}$ will come to rest. As a consequence, the $\eta^{\prime}$ will be strongly coupled to any collective flow of matter, and the $p_{T}$ distribution of $\eta^{\prime}$ may be strongly distorted relative to that in $p p$ collisions.

When the matter is at high energy density, there will be a mixing between the collective excitations which will become the $\eta$ and $\eta^{\prime}$ in the vacuum, so an enhancement of the $\eta^{\prime}$ will lead to an enhancement of the $\eta$ too. In addition, an important decay mode of the vacuum $\eta^{\prime}$ is into $\eta$ with a branching ratio of $65 \%$, leading to an enhancement of $\eta$ after the breakup of hadronic matter occurs.

There are several places where one might see the effects of the return of the ninth Goldstone boson. First, one might study low mass dileptons in the region above the $\pi^{0}$ Dalitz pairs and below the $\rho$. If the $\eta^{\prime} / \pi^{0}$ ratio is enhanced, there would be an enhancement due to the $\eta^{\prime} \rightarrow e^{+} e^{-} \gamma$ decay mode. In Fig. 1 we display the data as measured in the CERES experiment [25]; the paucity of dileptons in the mass region between the $\pi^{0}$ and the $\rho$ was also seen by the HELIOS experiment [26]. The contributions from measured and assumed abundances of $\pi^{0}, \eta, \rho, \omega, \eta^{\prime}$, and $\phi$ are shown explicitly taking into account the acceptance and resolution of the detector. In Fig. 2 we have scaled the computed $\eta^{\prime}$ contribution by 50 and 16 , corresponding to the ratios $\eta^{\prime} / \pi^{0}=1$ and 0.3 , respectively, where the latter value arises from taking into account the strange quark mass effects, see (19). To these were added the contributions from the other mesons, without change, from Fig. 1. With the enhancement factor of 50, the result is a little too big in the region between 50 and $250 \mathrm{MeV}$, exceeding two data points by about two standard deviations. Otherwise, the representation of the data is very good. With the enhancement factor of 16 , there is also a good representation, although the curve consistently falls below the data points by about one standard deviation between $350 \mathrm{MeV}$ and $850 \mathrm{MeV}$. We have made no attempt to compute the effects because of a changing shape of the $p_{T}$ spectrum caused by collective flow. Distortions of the $p_{T}$ spectrum folded into detection biases might have the effect of artificially enhancing or suppressing the $\eta^{\prime}$ contribution. Additional contributions come from dileptons produced in hadron-hadron collisions during the expansion and cooling phase, which help to fill in not only the mass region between $2 m_{\pi}$ and $m_{\rho}$ but also the region between the $\phi$ and the $J / \psi$ mesons [27].

We should caution the reader that a big enhancement of $\eta^{\prime}$ production would probably cause a suppression of direct production of other mesons due to energy conservation. For example, if the only mesons produced were the $\eta^{\prime}$ and the neutral and charged pions, and if $\eta^{\prime} / \pi^{0}$ was increased from 0.02 to 1 , then the total number of outcoming pions, includ-

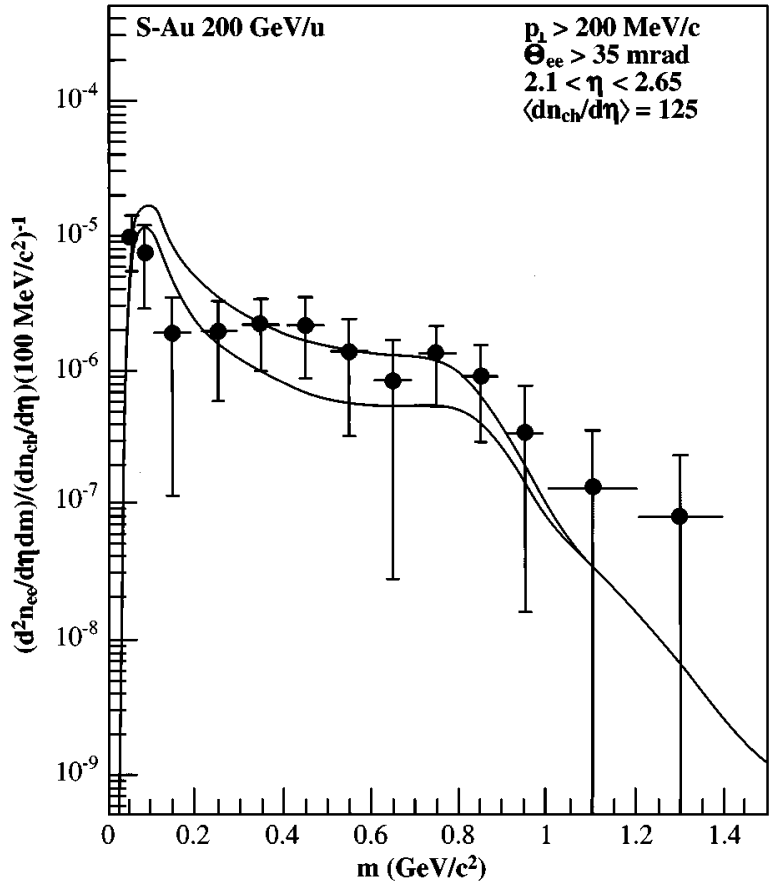

FIG. 2. The two curves are the result of multiplying the assumed $\eta^{\prime}$ constribution in Fig. 1 by factors of 16 and 50, and adding the other contributions.

ing those from $\eta^{\prime}$ decay, would approximately double. It would be a good exercise to refit the abundances of all the mesons with this effect taken into account. Of course, the total number of mesons could still increase, with the required energy coming from a decrease in the average momentum of the particles. This ties in with the problem of distortion of the $p_{T}$ spectrum due to collective flow.

Perhaps the most convincing demonstration of the return of the $\eta^{\prime}$ would be a direct measurement. This might be possible for the two-photon decay mode, especially if the production cross section is as strongly enhanced as we suggest. It would be important to have a simultaneous direct measurement of the $\eta$ since we expect an enhancement there too. In fact, some enhancement of the $\eta / \pi^{0}$ ratio in central $\mathrm{S}+\mathrm{Au}$ collisions was indeed observed experimentally by the WA80 experiment [28]. In minimum bias events, the ratio was measured to be $0.29 \pm 0.13$, consistent with protonproton collisions. In central collisions, the ratio was measured to be $0.54 \pm 0.14$. Both are integrated ratios from $p_{T}=$ 0 to $1 \mathrm{GeV} / c$. Since the branching ratio of $\eta^{\prime}$ into $\eta$ is about $65 \%$, an enhancement of $\eta^{\prime} / \pi^{0}=1$ is close to being ruled out (but recall the caveats about energy conservation and $p_{T}$ distortion mentioned above). An enhancement of $\eta^{\prime} / \pi^{0}$ $=0.3$ is more consistent with this data and more theoretically likely.

We should emphasize that unlike the case for the $\rho$ meson, and to a lesser degree for the $\omega$ and $\phi$, the $\eta^{\prime}$ and the $\eta$ mesons almost always decay after the surrounding hadronic matter has blown apart. Therefore, one cannot expect to directly see the effect of the mass shift of the $\eta^{\prime}$ or the 
$\eta$ meson: the only effect will be due to an enhanced production cross section.

Finally, we would like to comment on the effect that increased $\eta^{\prime}$ production will have on $\eta$ production, and its possible experimental consequences. If we assume that there is about a factor of 16 enhancement of $\eta^{\prime}$ production, this translates into an order 1 enhancement of $\eta$ production. Since the $\eta$ has a large branching mode into photons, a distortion of the photon spectra, or a direct measurement of the $\eta$ by its two-photon decay mode provides a signal. This would of course have to be in central collisions, and account would have to be taken of the possible distortion of the $\eta^{\prime}$ $p_{T}$ spectrum. It appears that such an enhancement is on the edge of current experimental detection and deserves closer study. On the other hand, if the $\eta^{\prime}$ production truly becomes of order 1 , then there is an enhancement of $\eta$ production by perhaps a factor of 3 . This appears to be ruled out in current experiments, but in the future could provide a test of this picture.

\section{ACKNOWLEDGMENTS}

We gratefully acknowledge conversations with D. Dyakonov, A. Efremov, H. Satz, E. Shuryak, R. Thews, I. Tserruya, X.-N. Wang, and I. Zahed. We thank Jean Tran Thanh Van and the organizers of the XXX Rencontres de Moriond where this work was initiated, and B. Müller, K. KinderGeiger, J.-Y. Ollitrault, and the ECT* in Trento, Italy for hospitality during the workshop on QCD and Ultrarelativistic Heavy Ion Collisions where this work was completed. This work was supported by the U.S. Department of Energy under Grant No. DE-FG02-87ER40328 and DE-FG02-94ER40823 and by the German Research Ministry (BMFT) under Contract No. 06 BI 721.
[1] J. Schwinger, Phys. Rev. 82, 664 (1951).

[2] S. L. Adler, Phys. Rev. 177, 2426 (1969).

[3] J. S. Bell and R. Jackiw, Nuovo Cimento A 60, 47 (1969).

[4] A. A. Belavin, A. M. Polyakov, A. S. Schwartz, and Yu. S. Tyupkin, Phys. Lett. 59B, 85 (1975).

[5] G. 't Hooft, Phys. Rev. Lett. 37, 8 (1976).

[6] D. J. Gross, R. D. Pisarski, and L. G. Yaffe, Rev. Mod. Phys. 53, 43 (1981).

[7] R. D. Pisarski and F. Wilczek, Phys. Rev. D 29, 338 (1984).

[8] T. Hashimoto, K. Hirose, T. Kanki, and O. Miyamura, Nucl. Phys. A478, 687c (1988).

[9] E. Shuryak, Comments Nucl. Part. Phys. 21, 235 (1994), and references therein.

[10] T. Hatsuda and T. Kunihiro, Phys. Rep. 247, 221 (1994), and references therein.

[11] D. I. Dyakonov and A. D. Mirlin, Phys. Lett. B 203, 299 (1988).

[12] E. Shuryak and M. Velkovsky, Phys. Rev. D 50, 3323 (1994).

[13] M. C. Chu and S. Schramm, Phys. Rev. D 51, 4580 (1995).

[14] E. Witten, Nucl. Phys. B156, 269 (1979).

[15] G. Veneziano, Nucl. Phys. B159, 213 (1979).

[16] J. Kogut and L. Susskind, Phys. Rev. D 11, 3594 (1975).

[17] D. I. Dyakonov and M. I. Eides, Sov. Phys. JETP 54, 232
(1981).

[18] G. Veneziano, Mod. Phys. Lett. A 4, 1605 (1989).

[19] A. V. Efremov, J. Soffer, and N. A. Törnqvist, Phys. Rev. Lett. 64, 1495 (1990).

[20] M. Bander, Phys. Rev. D 44, 3695 (1991).

[21] H. Gomm, Ö. Kaymakcalan, and J. Schechter, Phys. Rev. D 30, 2345 (1984).

[22] J. W. Durso, Phys. Lett. B 184, 348 (1987).

[23] Particle Data Group, Phys. Rev. D 50, Part 1 (1994).

[24] J. F. Donoghue, E. Golowich, and B. R. Holstein, Dynamics of the Standard Model (Cambridge University Press, Cambridge, England, 1992), Sec. VII-4.

[25] CERES Collaboration, G. Agakiechev et al., Report No. CERN-PPE/95-26, 1995 (unpublished); I. Tserruya, Nucl. Phys. A590, 127c (1995).

[26] HELIOS-3 Collaboration, M. Masera, Nucl. Phys. A590, 93c (1995).

[27] C. Gale and P. Lichard, Phys. Rev. D 49, 3338 (1994).

[28] WA80 Collaboration, A. Lebedev et al., in Quark Matter '93, Proceedings of the 10th International Conference on Ultrarelativistic Nucleus-Nucleus Collisions, Borlange, Sweden, edited by E. Stenlund et al. [Nucl. Phys. A566, 355c (1994)]. 